\title{
THE GROUP DETERMINANT DETERMINES THE GROUP
}

\author{
EDWARD FORMANEK AND DAVID SIBLEY
}

(Communicated by Warren J. Wong)

\begin{abstract}
Let $G=\left\{g_{1}, \ldots, g_{n}\right\}$ be a finite group of order $n$, let $K$ be a field whose characteristic is prime to $n$, and let $\left\{x_{g} \mid g \in G\right\}$ be independent commuting variables over $K$. The group determinant of $G$ is the determinant of the $n \times n$ matrix $\left(x_{g_{i} g_{j}^{-1}}\right)$. We show that two groups with the same group determinant are isomorphic.
\end{abstract}

\section{INTRODUCTION}

Let $G$ be a finite group of order $n$, let $\left\{x_{g} \mid g \in G\right\}$ be $n$ independent variables over a field $K$, and let $K\left(x_{g}\right)$ be the field of rational functions over $K$ in these variables. The group determinant, $\mathscr{D}_{G}=\mathscr{D}_{G}\left(x_{g}\right) \in K\left(x_{g}\right)$, is the determinant of the $n \times n$ matrix $\left(x_{g_{i} g_{j}^{-1}}\right)$, where $G=\left\{g_{1}, \ldots, g_{n}\right\}$. Equivalently, it is the determinant of the endomorphism of the group algebra $K\left(x_{g}\right) G$ induced by left multiplication by the "generic" element $\mathscr{X}_{G}=\Sigma x_{g} g$. The group determinant of $G$ is a homogeneous polynomial of degree $n$ with integer coefficients.

The representation theory of finite groups was developed by Frobenius in order to solve the problem of factoring the group determinant as an element of $\mathbb{C}\left[x_{g}\right]$, the polynomial ring over $\mathbb{C}$, the field of complex numbers. In modern language, his solution is as follows: The group algebra $\mathbb{C} G$ is isomorphic as a $\mathbb{C}$-algebra to a direct product of matrix algebras over $\mathbb{C}$. This isomorphism extends to an isomorphism of $\mathbb{C}\left(x_{g}\right)$-algebras:

$$
\rho=\left(\rho_{1}, \ldots, \rho_{k}\right): \mathbb{C}\left(x_{g}\right) G \rightarrow M_{m_{1}}\left(\mathbb{C}\left(x_{g}\right)\right) \times \cdots \times M_{m_{k}}\left(\mathbb{C}\left(x_{g}\right)\right) .
$$

If $z \in M_{m}(\mathbb{C})$ has determinant $\operatorname{det}(z)$ as an element of $M_{m}(\mathbb{C})$ (i.e., as an endomorphism of $\left.\mathbb{C}^{m}\right)$, then the determinant of the endomorphism of $M_{m}(\mathbb{C})$ defined by left multiplication by $z$ is $(\operatorname{det}(z))^{m}$, since as a left $M_{m}(\mathbb{C})$-module, $M_{m}(\mathbb{C})$ is isomorphic to $m$ copies of $\mathbb{C}^{m}$. This means that

$$
\mathscr{D}_{G}=\left(\operatorname{det}\left(\rho_{1}\left(\mathscr{X}_{G}\right)\right)\right)^{m_{1}} \cdots\left(\operatorname{det}\left(\rho_{k}\left(\mathscr{X}_{G}\right)\right)\right)^{m_{k}},
$$

and in fact this is a complete factorization of $\mathscr{D}_{G}$ in $\mathbb{C}\left[x_{g}\right]$.

Received by the editors March 29, 1990.

1980 Mathematics Subject Classification (1985 Revision). Primary 20C05.

Key words and phrases. Group determinant.

The first author was partially supported by the National Science Foundation. 
$\mathrm{K}$. W. Johnson [4, 5] has raised the question of whether the group determinant determines the group and explored connections with the work of Frobenius. He showed [5, Lemma 4.4] that if two groups have the same group determinant, then they form a Brauer pair, which means that they have the same character table and that there is a one-to-one correspondence between their conjugacy classes which preserves the $m$ th power map for every integer $m$. We thank him for sending us his work and a copy of a letter from H.-J. Hoehnke, who correctly suggested the relevance of a result of Frobenius closely related to Theorem 1 below.

Our main result, Theorem 5, is that the group determinant determines the group up to isomorphism. We show that if $G$ and $H$ are finite groups, $K$ is a field whose characteristic does not divide the order of $G$, and $\varphi: G \rightarrow H$ is a bijection of sets such that $\hat{\varphi}\left(\mathscr{D}_{G}\right)=\mathscr{D}_{H}$, where $\hat{\varphi}: K\left(x_{g}\right) \rightarrow K\left(x_{h}\right)$ is defined by $\hat{\varphi}\left(x_{g}\right)=x_{\varphi(g)}$, then $G$ is isomorphic to $H$. More precisely, if $\varphi$ satisfies the above hypotheses and $\psi: G \rightarrow H$ is defined by $\psi(g)=\varphi(g) \varphi(1)^{-1}$, then $\psi: G \rightarrow H$ is either an isomorphism or an anti-isomorphism. (An antihomomorphism is a map which reverses multiplication: $\psi(a b)=\psi(b) \psi(a)$.)

If $K$ has characteristic $p>0$, and the hypothesis that $p$ does not divide the order of $G$ is dropped, then we cannot expect the group determinant to determine the group. In the extreme case where $G$ is a $p$-group, the group determinant is $\left(\Sigma\left\{x_{g} \mid g \in G\right\}\right)^{|G|}$, which reflects only the order of $G$, and does not depend at all on the multiplication of $G$. We show that $\mathscr{D}_{G}$ is determined by $\mathscr{D}_{G / \mathscr{O}_{p}(G)}$ (Theorem 7), where $\mathscr{O}_{p}(G)$ is the largest normal $p$-subgroup of $G$, and that $\psi$ induces an isomorphism or anti-isomorphism between $G / \mathscr{O}_{p}(G)$ and $H / \mathscr{O}_{p}(H)$ (Theorem 6), which is all that could be hoped for in this case.

The proof is based on the following theorem of Dieudonne.

Theorem 1 (Dieudonne [1, Theoreme 3]). Let $K$ be a field, and let $\theta: M_{n}(K) \rightarrow$ $M_{n}(K)$ be an isomorphism of $K$-vector spaces which carries the set of matrices of determinant zero onto itself. Then $\theta$ is one of the following two possibilities.

(1) For all $X \in M_{n}(K), \theta(X)=A X B$, for fixed invertible $A, B \in M_{n}(K)$.

(2) For all $X \in M_{n}(K), \theta(X)=A X^{\mathrm{t}} B$, for fixed invertible $A, B \in M_{n}(K)$, where $X^{\mathrm{t}}$ is the transpose of $X$.

The theorem of Frobenius [3, p. 1011 (p. 99 in Gesammelte Abhandlungen, Band III)] cited by Hoehnke has the same conclusion as Theorem 1, but with the apparently stronger hypothesis that $\theta$ preserves the determinant up to multiplication by a fixed scalar. It is also proved for the field of complex numbers only. Over the complex field, Dieudonne's theorem can be deduced from the theorem of Frobenius using the Nullstellensatz, and the fact that the determinant of an $n \times n$ matrix, regarded as a polynomial in $n^{2}$ variables, is irreducible.

For any ring $R$, let $\mathscr{U}(R)$ denote the group of units of $R$. Of course, $\mathscr{U}\left(M_{n}(K)\right)$ is just the set of matrices with nonzero determinant. For our pur- 
poses, it is convenient to use the following variant of Theorem 1 .

Corollary 2. Let $\theta: M_{n}(K) \rightarrow M_{n}(K)$ be an isomorphism of $K$-vector spaces. Suppose that $\theta\left(\mathscr{U}\left(M_{n}(K)\right)=\mathscr{U}\left(M_{n}(K)\right)\right.$ and $\theta(1)=1$. Then $\theta$ is either a ring isomorphism or a ring anti-isomorphism.

Section 2 of our paper generalizes Corollary 2 to direct products of matrix rings (Theorem 4). In $\S 3$ we combine this extension with some observations about groups and their representations to derive our main theorem. In $\S 4$ we indicate how to deal with the case where the characteristic of the field $K$ divides the order of the group $G$.

\section{Generalizing the theorem of Dieudonne to DiRect PRODUCts}

Lemma 3. Let $K$ be an infinite field, let $S=S_{1} \times \cdots \times S_{l}$, where $S_{i}=M_{n_{i}}(K)$, and let $W$ be a $K$-vector subspace of $S$.

(A) If $W$ consists of nonunits, then there is an index $j$, where $1 \leq j \leq l$, such that $\operatorname{det}\left(w_{j}\right)=0$ for all $w=\left(w_{1}, \ldots, w_{l}\right) \in W$.

(B) If $W$ consists of nonunits, and is maximal with respect to this property (in the sense that if $W \subseteq W^{\prime} \subseteq S$, and $W \neq W^{\prime}$, then $W^{\prime}$ contains a unit), then there is an index $j$ such that

$$
W=S_{1} \times \cdots \times S_{j-1} \times W_{j} \times S_{j+1} \times \cdots \times S_{l},
$$

where $W_{j}$ is a maximal $K$-vector subspace of $S_{j}=M_{n_{j}}(K)$ consisting of nonunits.

Proof. (A) Suppose there is no such index $j$. We show by induction on $i$ that for $i=1, \ldots, l$, there is some $w=\left(w_{1}, \ldots, w_{l}\right) \in W$ with $\operatorname{det}\left(w_{1}\right) \cdots \operatorname{det}\left(w_{i}\right)$ $\neq 0$, which shows (when $i=l$ ) that $W$ contains a unit.

For $i=1$, choose $w=\left(w_{1}, \ldots, w_{l}\right) \in W$ with $\operatorname{det}\left(w_{1}\right) \neq 0$.

For the inductive step, assume that $w=\left(w_{1}, \ldots, w_{l}\right) \in W$, and that $\operatorname{det}\left(w_{1}\right) \cdots \operatorname{det}\left(w_{i-1}\right) \neq 0$, and choose $v=\left(v_{1}, \ldots, v_{l}\right) \in W$ with $\operatorname{det}\left(v_{i}\right) \neq 0$. If $\alpha$ is a nonzero element of $K$, then $\operatorname{det}\left(w_{e}-\alpha v_{e}\right) \neq 0(e=1, \ldots, i-1)$ unless $\alpha^{-1}$ is an eigenvalue of $w_{e}^{-1} v_{e}$, and $\operatorname{det}\left(w_{i}-\alpha v_{i}\right) \neq 0$ unless $\alpha$ is an eigenvalue of $w_{i} v_{i}^{-1}$. Since the set of such $\alpha$ is finite, we can choose $\beta \in K$ such that $\operatorname{det}\left(w_{1}-\beta v_{1}\right) \cdots \operatorname{det}\left(w_{i}-\beta v_{i}\right) \neq 0$, where $w-\beta v=\left(w_{1}-\beta v_{1}, \ldots, w_{l}-\beta v_{l}\right) \in$ $W$, which completes the inductive step.

(B) is an immediate corollary of (A).

Theorem 4. Let $K$ be an infinite field, let $R=R_{1} \times \cdots \times R_{k}$, where $R_{i}=$ $M_{m_{i}}(K)$, and let $S=S_{1} \times \cdots \times S_{l}$, where $S_{j}=M_{n_{j}}(K)$. Suppose that $\theta: R \rightarrow S$ is an isomorphism of $K$-vector spaces and $\theta(\mathscr{U}(R))=\mathscr{U}(S)$.

(A) Then $k=l$, and there is a permutation $\pi$ of $1, \ldots, k$ such that $\theta\left(R_{i}\right)=$ $S_{\pi(i)}$ for $i=1, \ldots, k$.

(B) If $\theta(1)=1$, each induced map $\theta: R_{i} \rightarrow S_{\pi(i)}$ is either a ring isomorphism or a ring anti-isomorphism (depending on $i$ ). 
Proof. (A) For each integer $i$, where $1 \leq i \leq k$, let $K^{m_{i}}=\left\{\left(\alpha_{1}, \ldots, \alpha_{m_{i}}\right) \mid \alpha_{d} \in\right.$ $K\}$, and let $R_{i}=M_{m_{i}}(K)$ act on $K^{m_{i}}$ by right multiplication. For each nonzero $\alpha \in K^{m_{i}}$, set $V_{\alpha}=\left\{r \in R_{i} \mid \alpha r=0\right\}$. Then

$$
R_{1} \times \cdots \times R_{i-1} \times V_{\alpha} \times R_{i+1} \times \cdots \times R_{k}
$$

is a maximal $K$-vector subspace of $R$ consisting of nonunits, so its image under $\theta$ is a maximal $K$-vector subspace of $S$ consisting of nonunits. By Lemma $3(\mathrm{~B})$, there is an index $j(\alpha)$ (apparently depending on $\alpha$ ) such that

$$
\theta\left(R_{1} \times \cdots \times V_{\alpha} \times \cdots \times R_{k}\right)=S_{1} \times \cdots \times S_{j(\alpha)-1} \times W_{\alpha} \times S_{j(\alpha)+1} \times \cdots \times S_{l},
$$

where $W_{\alpha}$ is a maximal $K$-vector subspace of $S_{j(\alpha)}$ consisting of nonunits.

We claim that $j(\alpha)=j(\beta)$ for all nonzero $\alpha, \beta \in K^{m_{1}}$. For suppose that $j(\alpha) \neq j(\beta)$ for some $\alpha, \beta$. Then, since $V_{\alpha+\beta} \supseteq V_{\alpha} \cap V_{\beta}$,

$$
\theta\left(R_{1} \times \cdots \times V_{\alpha+\beta} \times \cdots \times R_{k}\right) \supseteq S_{1} \times \cdots \times W_{\alpha} \times \cdots \times W_{\beta} \times \cdots \times S_{l} .
$$

Since $\theta\left(R_{1} \times \cdots \times V_{\alpha+\beta} \times \cdots \times R_{k}\right)$ has the form specified by Lemma 3(B), (1) implies that it is equal to either $S_{1} \times \cdots \times W_{\alpha} \times \cdots \times S_{l}$ or $S_{1} \times \cdots \times W_{\beta} \times \cdots \times S_{l}$. Thus $V_{\alpha+\beta}=V_{\alpha}$ or $V_{\alpha+\beta}=V_{\beta}$, which forces $V_{\alpha}=V_{\beta}=V_{\alpha+\beta}$, and $j(\alpha)=$ $j(\beta)$.

Let $\pi(i)$ denote the common value of $j(\alpha)$ for nonzero $\alpha \in K^{m_{i}}$. Since $\bigcap\left\{V_{\alpha} \mid \alpha \in K^{m_{i}}, \alpha \neq 0\right\}=0$,

$$
\theta\left(R_{1} \times \cdots \times \underset{i}{0} \times \cdots \times R_{k}\right) \supseteq S_{1} \times \cdots \times \underset{\pi(i)}{0} \times \cdots \times S_{l} .
$$

Applying the same argument to $\theta^{-1}$, there is an index $e$ such that

$$
\theta^{-1}\left(S_{1} \times \cdots \times \underset{\pi(i)}{0} \times \cdots \times S_{l}\right) \supseteq R_{1} \times \cdots \times \underbrace{0}_{e} \times \cdots \times R_{k} .
$$

Combining (2) and (3) implies that $i=e$ and that there is equality in (2). It follows easily that $k=l$ and $\pi:\{1, \ldots, k\} \rightarrow\{1, \ldots, l\}$ is a bijection such that $\theta\left(R_{i}\right)=S_{-\pi(i)}$.

(B) is an immediate consequence of (A) and Corollary 2.

\section{Proof of THE MAIN THEOREM}

Theorem 5. Let $G$ and $H$ be finite groups, with group determinants $\mathscr{D}_{G}=$ $\mathscr{D}_{G}\left(x_{g}\right) \in K\left(x_{g}\right), \mathscr{D}_{H}=\mathscr{D}_{H}\left(x_{h}\right) \in K\left(x_{h}\right)$, where $K$ is a field whose characteristic does not divide the order of $G$. Let $\varphi: G \rightarrow H$ be a bijection of sets, and let $\hat{\varphi}: K\left(x_{g}\right) \rightarrow K\left(x_{h}\right)$ be the field isomorphism induced by $\hat{\varphi}\left(x_{g}\right)=x_{\varphi(g)}$. Suppose that $\hat{\varphi}\left(\mathscr{D}_{G}\left(x_{g}\right)\right)=\mathscr{D}_{H}\left(x_{h}\right)$. Then the map $\psi: G \rightarrow H$ defined by $\psi(g)=\varphi(g) \varphi(1)^{-1}$ is either a group isomorphism or a group anti-isomorphism. In particular, if $\varphi(1)=1$, then $\varphi$ is a group isomorphism or a group antiisomorphism. In any case, $G$ is isomorphic to $H$, since any group is antiisomorphic to itself.

Proof. Let $\Delta_{G}(\alpha)\left(\Delta_{H}(\beta)\right)$ be the determinant of the endomorphism of $K G$ $(K H)$ induced by left multiplication by $\alpha \in K G(\beta \in K H)$. By definition, 
$\mathscr{D}_{G}\left(x_{g}\right)=\Delta_{G}\left(\mathscr{X}_{G}\right)=\Delta_{G}\left(\Sigma x_{g} g\right), \mathscr{D}_{H}\left(x_{h}\right)=\Delta_{H}\left(\mathscr{X}_{H}\right)=\Delta_{H}\left(\Sigma x_{h} h\right)$. For arbitrary $\alpha=\Sigma \alpha_{g} g \in K G, \beta=\Sigma \beta_{h} h \in K H$, it is clear that

$$
\begin{aligned}
\Delta_{G}(\alpha) & =\mathscr{D}_{G}\left(\alpha_{g}\right)=\mathscr{D}_{G}\left(x_{g} \mid x_{g}=\alpha_{g}\right), \\
\Delta_{H}(\beta) & =\mathscr{D}_{H}\left(\beta_{h}\right)=\mathscr{D}_{H}\left(x_{h} \mid x_{h}=\beta_{h}\right) .
\end{aligned}
$$

Extend $\varphi$ to a $K$-vector space isomorphism $\varphi: K G \rightarrow K H$ by

$$
\varphi\left(\Sigma\left\{\alpha_{g} g \mid g \in G\right\}\right)=\Sigma\left\{\alpha_{g} \varphi(g) \mid g \in G\right\}=\Sigma\left\{\alpha_{\varphi^{-1}(h)} h \mid h \in H\right\} .
$$

Since $\mathscr{D}_{G}\left(x_{\varphi(g)}\right)=\hat{\varphi}\left(\mathscr{D}_{G}\left(x_{g}\right)=\mathscr{D}_{H}\left(x_{h}\right)\right.$ by hypothesis,

$$
\begin{aligned}
\Delta_{G}(\alpha) & =\mathscr{D}_{G}\left(x_{g} \mid x_{g}=\alpha_{g}\right)=\mathscr{D}_{G}\left(x_{\varphi(g)} \mid x_{\varphi(g)}=\alpha_{g}\right) \\
& =\mathscr{D}_{H}\left(x_{h} \mid x_{h}=\alpha_{\varphi^{-1}(h)}\right)=\Delta_{H}(\varphi(\alpha)),
\end{aligned}
$$

for any $\alpha=\Sigma \alpha_{g} g \in K G$. Note that $\alpha \in K G$ is a unit if and only if $\Delta_{G}(\alpha) \neq 0$, and $\beta \in K H$ is a unit if and only if $\Delta_{H}(\beta) \neq 0$. Hence $(*)$ implies that $\varphi(\mathscr{U}(K G))=\mathscr{U}(K H)$. Extend $\psi: G \rightarrow H$ to a $K$-vector space isomorphism $\psi: K G \rightarrow K H$ by $\psi(\alpha)=\varphi(\alpha) \varphi(1)^{-1}$. Then $\psi(\mathscr{U}(K G))=\mathscr{U}(K H)$ and $\psi(1)=1$.

The group determinant is unchanged if the field $K$ is enlarged, so we may assume that $K$ is algebraically closed. Then $K$ is infinite and $K G$ and $K H$ are direct products of matrix rings over $K$. Take $K$-algebra isomorphisms

$$
\begin{aligned}
& \rho=\left(\rho_{1}, \ldots, \rho_{k}\right): K G \rightarrow R=R_{1} \times \cdots \times R_{k}, \quad \text { where } R_{i}=M_{m_{i}}(K), \\
& \sigma=\left(\sigma_{1}, \ldots, \sigma_{l}\right): K H \rightarrow S=S_{1} \times \cdots \times S_{l}, \quad \text { where } S_{j}=M_{n_{j}}(K),
\end{aligned}
$$

and let $\theta=\sigma \psi \rho^{-1}: R \rightarrow S$.

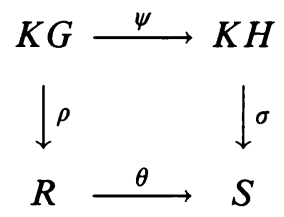

Since $\rho$ and $\sigma$ are ring isomorphisms, $\theta(\mathscr{U}(R))=\mathscr{U}(S)$ and $\theta(1)=1$. Theorem 4 applies to $\theta$, so $k=l$, and after permuting the factors of $S$, we may suppose that for each $i, \theta\left(R_{i}\right)=S_{i}$, and $\theta: R_{i} \rightarrow S_{i}$ is either a ring isomorphism or a ring anti-isomorphism (depending on $i$ ). Set

$$
\begin{aligned}
& R^{+}=\Pi\left\{R_{i} \mid \theta: R_{i} \rightarrow S_{i} \text { is an isomorphism }\right\}, \\
& R^{-}=\Pi\left\{R_{i} \mid \theta: R_{i} \rightarrow S_{i} \text { is an anti-isomorphism }\right\} .
\end{aligned}
$$

(If $R_{i}=K=S_{i}$, then $\theta: R_{i} \rightarrow S_{i}$ is both an isomorphism and an antiisomorphism, and we include $R_{i}$ in both $R^{+}$and $R^{-}$. We define $S^{+}$and $S^{-}$ in the obvious way.)

The compositions

$$
\begin{aligned}
& G \rightarrow K G \stackrel{\rho}{\rightarrow} R \rightarrow R^{+} \stackrel{\theta}{\rightarrow} S^{+} \\
& G \rightarrow K G \stackrel{\rho}{\rightarrow} R \rightarrow R^{-} \stackrel{\theta}{\rightarrow} S^{-}
\end{aligned}
$$


are, respectively, multiplicative and antimultiplicative. Let $\mathrm{N}^{+}$and $\mathrm{N}^{-}$denote their kernels (the group elements sent to 1). They are normal subgroups of $G$ whose intersection is trivial, since $G$ embeds in $K G$. Thus $N^{+}$and $N^{-}$ centralize each other, so their direct product $N^{+} \times N^{-}$is a subgroup of $G$.

We claim that $N^{+}=1$ or $N^{-}=1$. Suppose not, and let $\mu: N^{+} \rightarrow M_{q}(K)$, $\nu: N^{-} \rightarrow M_{r}(K)$ be nontrivial irreducible representations. Then $\mu \otimes \nu: N^{+} \times$ $N^{-} \rightarrow M_{q}(K) \otimes M_{r}(K) \approx M_{q r}(K)$ is an irreducible representation of $N^{+} \times N^{-}$, and neither $N^{+}$nor $N^{-}$is contained in the kernel of $\mu \otimes \nu$. There exists some irreducible representation $\rho_{j}: G \rightarrow M_{m_{j}}(\mathbb{C})=R_{j}$ whose restriction to $N^{+} \times N^{-}$ contains $\mu \otimes \nu$ as a component. Such a representation $\rho_{j}$ contains neither $N^{+}$ nor $N^{-}$in its kernel. But the definition of $N^{+}$and $N^{-}$above shows that every $\rho_{i}$ contains either $N^{+}$or $N^{-}$in its kernel, depending on whether $\theta: R_{i} \rightarrow S_{i}$ is an isomorphism or an anti-isomorphism. This contradiction proves our claim, so $N^{+}=1$ or $N^{-}=1$.

If $N^{+}=1$, then $\psi: G \rightarrow H$ is a group isomorphism, and if $N^{-}=1$, it is a group anti-isomorphism.

\section{WHAT HAPPENS WHEN THE CHARACTERISTIC OF $K$ DIVIDES THE ORDER OF $G$ ?}

Let $K$ be a field of characteristic $p>0$. Assume all the hypotheses and notation of Theorem 5, excepting the hypothesis that $p$ does not divide the order of $G$. The initial steps in the proof of Theorem 5 remain valid, and the $K$-vector space isomorphism $\psi: K G \rightarrow K H$ still satisfies $\psi(1)=1$, and

$$
\psi(\mathscr{U}(K G))=\mathscr{U}(K H) .
$$

There is also no loss of generality in assuming that $K$ is algebraically closed.

At this point we must pay heed to the fact that if $p$ divides the order of $G$, then $K G$ and $K H$ are not direct products of matrix rings over $K$. They have Jacobson radicals $\mathscr{J} K G$ and $\mathscr{J} K H$, and $K G / \mathscr{J} K G$ and $K H / \mathscr{J} K H$ are direct products of matrix rings over $K$.

If $y \in \mathscr{J} K G$, then $\mathscr{U}(K G)+y=\mathscr{U}(K G)$, for this is one of the basic properties of the Jacobson radical. Conversely, if $y \notin \mathscr{J} K G$, then (because $K G / \mathscr{J} K G$ is a product of matrix rings over $K)$ there is a $u \in \mathscr{U}(K G)$ such that $u+y \notin \mathscr{U}(K G)$. Hence

$$
\begin{aligned}
& \mathscr{J} K G=\{y \in K G \mid \mathscr{U}(K G)+y=\mathscr{U}(K G)\}, \\
& \mathscr{J} K H=\{y \in K H \mid \mathscr{U}(K H)+y=\mathscr{U}(K H)\} .
\end{aligned}
$$

Since $\psi$ is a linear map, (4) and (5) imply that $\psi(\mathscr{J} K G)=\mathscr{J} K H$. Thus $\psi$ induces a $K$-vector space isomorphism $\psi^{*}: K G / \mathscr{J} K G \rightarrow K H / \mathscr{J} K H$, and $\psi^{*}$ preserves units, by (4) and (5). Because $K G / \mathscr{J} K G$ and $K H / \mathscr{J} K H$ are direct products of matrix rings over $K$, Theorem 4 implies that $\psi^{*}$ carries simple factors to simple factors, and is either an isomorphism or an anti-isomorphism on each simple factor. 
A slight elaboration of the corresponding argument in the proof of Theorem 5 shows that either $\psi^{*}$ is an isomorphism on every simple factor, or an antiisomorphism on every simple factor. Thus $\psi^{*}$ is either a ring isomorphism or a ring anti-isomorphism.

Consider the commutative diagram

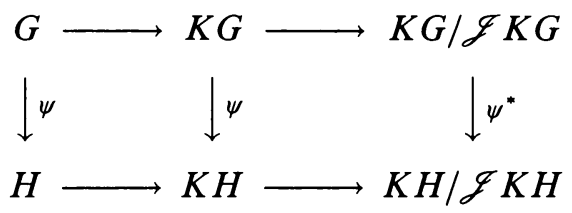

The largest normal $p$-subgroup of $G$ is denoted $\mathscr{O}_{p}(G)$. It is the kernel of the composition $G \rightarrow K G \rightarrow K G / \mathcal{J} K G$ [2, Corollary 2.13(ii), p. 102]. Thus (3) implies that $\psi^{*}: G / \mathscr{O}_{p}(G) \rightarrow H / \mathscr{O}_{p}(H)$ is either a group isomorphism or a group anti-isomorphism, depending on whether $\psi^{*}$ is a ring isomorphism or a ring anti-isomorphism. This yields

Theorem 6. Retain the hypotheses and notation of Theorem 5, except omit the hypothesis that $p$ does not divide the order of $G$, where $K$ has characteristic $p>0$. Then the map $\psi: G \rightarrow H$ defined by $\psi(g)=\varphi(g) \varphi(1)^{-1}$ induces either a group isomorphism or a group anti-isomorphism between $G / \mathscr{O}_{p}(G)$ and $H / \mathscr{O}_{p}(H)$.

Finally, we indicate how $\mathscr{D}_{G}$ can be expressed in terms of $\mathscr{D}_{G / \mathscr{O}_{p}(G)}$. We assume that $K$ is algebraically closed of characteristic $p>0$. The two observations required are

(I) If the regular representation of $G$ is conjugated into block upper triangular form, then the diagonal blocks are irreducible representations of $G$ and hence contain $\mathscr{O}_{p}(G)$ in their kernel [2, Corollary 2.13(ii), p. 102]. In particular, $G$ and $G / \mathscr{O}_{p}(G)$ have the same irreducible representations.

(II) The number of diagonal blocks corresponding to a given irreducible representation $\rho$ of $G$ in the regular representation of $G$ (conjugated into block upper triangular form) is $\left|\mathscr{O}_{p}(G)\right|$ times the number of blocks corresponding to $\rho$ in the regular representation of $G / \mathscr{O}_{p}(G)$ (conjugated into block upper triangular form).

We omit a proof of (II) since it requires a lot of notation from modular representation theory. The key fact it depends on is [2, Lemma 4.26, p. 162]. Our final result follows easily from (I) and (II).

Theorem 7. Let $K$ be a field of characteristic $p>0$. Let $\mathscr{D}_{G / \mathscr{O}_{p}(G)}=\mathscr{D}_{G / \mathscr{O}_{p}(G)}\left(x_{\bar{g}}\right)$, and for each $\bar{g}=\mathscr{O}_{p}(G) g \in G / \mathscr{O}_{p}(G)$, let $y_{\bar{g}}=\Sigma\left\{x_{h} \mid h \in \mathscr{O}_{p}(G) g\right\}$. Then $\mathscr{D}_{G}\left(x_{h}\right)=\left[\mathscr{D}_{G / \mathscr{O}_{p}(G)}\left(y_{\bar{g}}\right)\right]^{\left|\mathscr{O}_{p}(G)\right|}$. 


\section{REFERENCES}

1. J. Dieudonne, Sur une generalisation du groupe orthogonal a quatre variables, Arch. Math. 1 (1949), 282-287.

2. W. Feit, The representation theory of finite groups, North-Holland, Amsterdam, 1982.

3. F. G. Frobenius, Über die Darstellung der endlichen Gruppen durch lineare substitutionen, Sitz. Kön. Preuss. Akad. Wiss. Berlin (1897), 944-1015; Band III of F. G. FrobeniusGesammelte Abhandlungen, Springer-Verlag, Berlin, 1968, pp. 82-118.

4. K. W. Johnson, Latin square determinants, Algebraic, Extremal, and Metric Combinatorics (M.-M. Deza, P. Frankl, and I. G. Rosenberg, eds.), pp. 146-154; London Math. Soc. Lecture Notes, No. 131, Cambridge Univ. Press, Cambridge, 1988.

5. _ On the group determinant, preprint.

Department of Mathematics, Pennsylvania State University, University Park, PennSYLVANIA 16802 